\title{
A (giant) void is not mandatory to explain away dark energy with a Lemaître-Tolman model
}

\author{
M.-N. Célérier ${ }^{1}$, K. Bolejko ${ }^{2,3}$, and A. Krasiński ${ }^{3}$ \\ 1 Laboratoire Univers et Théories (LUTH), Observatoire de Paris, CNRS, Université Paris-Diderot, 5 place Jules Janssen, \\ 92190 Meudon, France \\ e-mail: marie-noelle.celerier@obspm.fr \\ 2 Department of Mathematics and Applied Mathematics, University of Cape Town, Rondebosch 7701, South Africa \\ 3 Nicolaus Copernicus Astronomical Centre, Polish Academy of Sciences, Bartycka 18, 00716 Warszawa, Poland
}

Received 2 November 2009 / Accepted 3 May 2010

\section{ABSTRACT}

\begin{abstract}
Context. Lemaitre-Tolman (L-T) toy models with a central observer have been used to study the effect of large scale inhomogeneities on the SN Ia dimming. Claims that a giant void is mandatory to explain away dark energy in this framework are currently dominating. Aims. Our aim is to show that L-T models exist that reproduce a few features of the $\Lambda$ CDM model, but do not contain the giant cosmic void.

Methods. We propose to use two sets of data - the angular diameter distance together with the redshift-space mass-density and the angular diameter distance together with the expansion rate - both defined on the past null cone as functions of the redshift. We assume that these functions are of the same form as in the $\Lambda$ CDM model. Using the Mustapha-Hellaby-Ellis algorithm, we numerically transform these initial data into the usual two L-T arbitrary functions and solve the evolution equation to calculate the mass distribution in spacetime.

Results. For both models, we find that the current density profile does not exhibit a giant void, but rather a giant hump. However, this hump is not directly observable, since it is in a spacelike relation to a present observer.

Conclusions. The alleged existence of the giant void was a consequence of the L-T models used earlier because their generality was limited a priori by needless simplifying assumptions, like, for example, the bang-time function being constant. Instead, one can feed any mass distribution or expansion rate history on the past light cone as initial data to the L-T evolution equation. When a fully general L-T metric is used, the giant void is not implied.
\end{abstract}

Key words. cosmology: dark energy - cosmology: miscellaneous

\section{The historical background of the problem}

In the framework of the homogeneous and isotropic standard cosmological model, the dimming of the type Ia supernovae as compared to their expected luminosity in an Einstein-de Sitter model is interpreted as a consequence of an assumed accelerated expansion of the Universe. This leads to the widespread belief in a "dark energy" component currently dominating the energy budget of our Universe. But this is not the only possible explanation of the SN Ia observations.

Shortly after the discovery by Riess et al. (1998) and Perlmutter et al. (1999) of the supernova dimming, it was proposed by several authors that this effect could be due to the largescale inhomogeneities (Pascual-Sánchez 1999; Célérier 2000; Tomita 2000, 2001a,b). After a period of relative disaffection, this proposal experienced a renewed interest about five years ago.

Three methods have been used to implement such a proposal: computation of backreaction terms in the dynamical equations using an averaging procedure proposed by Buchert (2000, 2001), calculations in the framework of a perturbative scheme and the use of exact inhomogeneous models, in particular those of Lemaître (1933) - Tolman (1934) (L-T) (see Célérier 2007, for a review).

The L-T model became rapidly popular for the purpose of mimicking "dark energy" because it exhibits three interesting features: i) it is one of the few exact solutions of General Relativity able to represent a physically consistent model of the matter dominated era of the Universe; ii) among these few, it is the most easily tractable from a computational point of view; iii) it is not an alternative to, but a generalisation of the Friedmann dust models (which are contained in it as a subcase), so can reproduce all the Friedmann-based results, including those of the "concordance" $\Lambda \mathrm{CDM}$ model, with an arbitrary precision. For more on this, in relation to the main subject of this paper, see the last section.

Three classes of models have been constructed with the L-T solution: i) models where the observer is located at the centre of a single L-T universe (e.g., Iguchi et al. 2002; Alnes et al. 2006; Apostolopoulos et al. 2006; Bolejko 2008; Garcia-Bellido \& Haugbølle 2008a, 2009); ii) models where the observer is located off the centre of such a universe (e.g., Schneider \& Célérier 1999; Apostolopoulos et al. 2006; Alnes \& Amarzguioui 2007) ${ }^{1}$; iii) Swiss-cheese models where the holes are L-T bubbles carved out of a Friedmannian homogeneous background (e.g., Brouzakis et al. 2007, 2008; Biswas \& Notari 2008; Marra et al. 2007).

\footnotetext{
${ }^{1}$ However, these authors have shown that the CMB data put very stringent limits on the distance of the observer from the centre of the model or/and on the amplitude of the inhomogeneities in an off-centre observer model.
} 
As will be recalled in Sect. 2, an L-T model is defined by two independent arbitrary functions of the radial coordinate, which can be fitted to the observational data. However, in most of the models currently available in the literature, the authors have artificially limited the generality by giving the L-T initial-data functions a handpicked algebraic form (depending on the authors' feelings about which kind of model would best represent our Universe), with only a few constant parameters being left arbitrary - to be adapted to the observations.

Another way in which the generality of the L-T models was artificially limited was the assumption that the age of the Universe is everywhere the same, i.e. that the L-T bang-time function $t_{\mathrm{B}}$ is constant. With $t_{\mathrm{B}}$ being constant, the only singlepatch L-T model that fits observations is one with a giant void (Iguchi et al. 2002; Yoo et al. 2008). Conceptually there is nothing wrong with a non-simultaneous big bang (even though this is a radical qualitative difference with the FLRW models), but one should exercise caution when referring to $\left(t-t_{\mathrm{B}}\right)$ as the actual age of the Universe. The L-T model is too simple to extend it up to instants earlier than decoupling. Therefore $t_{\mathrm{B}}$ should merely be regarded as a function that describes a degree of inhomogeneity of the initial conditions rather than as the actual instant of birth of the Universe.

The argument brought in defense of the constant $t_{\mathrm{B}}$ assumption is this: a non-constant $t_{\mathrm{B}}$ generates decreasing modes of perturbation of the metric (Silk 1977; Plebański \& Krasiński 2006), so any substantial inhomogeneity at the present time stemming from $t_{\mathrm{B}, r} \neq 0$ would imply "huge" perturbations of homogeneity at the last scattering. This, in turn, would contradict the CMB observations and the implications of inflationary models (we deliberately do not give references here, to avoid blaming any single individual for what seems to be a piece of conventional wisdom). However, these are only expectations that should not be treated as objective truth until they are verified by calculations. Such calculations have already been done, and it turned out that the inhomogeneities in $t_{\mathrm{B}}$ needed to explain the formation of galaxy clusters and voids are of the order of a few hundred years (Bolejko et al. 2005; Krasiński \& Hellaby 2004; Bolejko 2009). Then, on the basis of Bolejko's (2009) models 4 and 5, one can calculate that for a structure of present radius $30 \mathrm{Mpc}$ this age difference between the oldest and youngest region would generate $\mathrm{CMB}$ temperature fluctuations equal to $\Delta T / T=3.44 \times 10^{-6}$ and $\Delta T / T=-2.35 \times 10^{-6}$, respectively. This is well-hidden in the observational errors at the present level of precision. (In the future, when, presumably, the precision will improve, these results may possibly be used to measure the gradient of $t_{\mathrm{B}}$.) So there is no observational justification to the assumption $t_{\mathrm{B}}=$ constant.

These are the reasons why, in the recent years, we have seen the increase in popularity of void models, where the observer is located at or near the centre of a large, huge, giant L-T void of size of up to a few Gpc. Many authors have constructed classes of L-T models with a central local void and have shown that they were able to fit the SN Ia and other cosmological data provided the void is large (e.g., it has a diameter of $400 \mathrm{~h}^{-1} \mathrm{Mpc}$ in Alexander et al. 2009) or even huge (e.g., $1.35 \mathrm{Gpc}$ in Alnes et al. 2006, >2 Gpc in Garcia-Bellido \& Haugbølle 2008a and 2009), depending on the features of the particular model they had chosen. This contributed to the spreading of the belief in the necessity of a "giant local void" to resolve the "cosmological constant problem" with L-T models. However, as shown by Mustapha et al. (1998) and used as an illustration for the application to the supernova data and the "cosmological constant problem" by Célérier (2000), a given set of isotropic data can constrain only one of the two free functions of an L-T model and therefore, after fitting the supernova data with a given L-T solution, we are left with plenty of room to accommodate more observations.

Actually, a few authors discarded the central void hypothesis and proposed models with no such void (e.g., Iguchi et al. 2002). Enqvist \& Mattsson (2007) even showed that the fitting of the SN Ia data can be better with L-T models where the density distribution is constant on a constant-time hypersurface than with the $\Lambda$ CDM model (see also Bolejko 2008; Bolejko \& Wyithe 2009). Even though such a density distribution is not what is actually observed at very large scales by astronomers (it is not even observable, being in a spacelike relation to the central observer), the $\rho\left(t_{0}, r\right)=$ const. configuration vividly illustrates how misleading the FLRW-based geometrical intuitions can be. A spatial distribution of matter can radically change with time in consequence of an inhomogeneous expansion distribution in space. Our models will provide more examples of this phenomenon, and we will come back to this point in the conclusion.

Our aim here is to show, using two explicit examples reproducing the observational features of the $\Lambda$ CDM model, that a giant void is not at all a necessary implication of using L-T models. We propose to use input functions that can be derived from observations ${ }^{2}$. Our L-T toy models will be constrained by the angular diameter distance together with the redshift-space mass-density or the angular diameter distance together with the expansion rate.

It should be noted that these functions have not the same form in the $\Lambda \mathrm{CDM}$ model and in giant-void L-T models. For example, let us consider the giant void model from Bolejko \& Wyithe (2009) with radius of $2.96 \mathrm{Gpc}$ and density contrast of 4.05. The redshift-space mass-density for this model and for the $\Lambda \mathrm{CDM}$ model are shown in Fig. 1 . As seen, at $z \approx 1$ the difference between these two models is more than a factor of 2 . Also, the expansion rate as a function of redshift behaves differently (for details and constraints coming from $H(z)$ see Bolejko \& Wyithe 2009). Thus giant void models have difficulties to mimic all the observational features of the dark energy model (Zibin et al. 2008; Clifton et al. 2009).

In this paper, we show that if the observational data are properly fitted to these $\Lambda \mathrm{CDM}$ functions, then a giant void is not mandatory to explain them. In fact the L-T models that mimic our choice of observational features of the $\Lambda$ CDM model have a central Gpc-scale overdensity rather than an underdensity. We emphasise that what we reproduce in our L-T model are not the actual observational relations, but the parameters of the $\Lambda \mathrm{CDM}$ model fitted to the observations - which is not the same thing.

Note that this model is not designed to reproduce all the available cosmological data, nor is it to be considered as the final model of our Universe. Its purpose is to exemplify the proper use of L-T models and to show what can come out of it. Moreover, it should be understood as tentative beyond the redshift range in which the $\Lambda \mathrm{CDM}$ functions we use are robustly established.

\footnotetext{
2 One should be aware that there is a great deal of phenomenology involved in interpreting the observations. For example, with supernovae, the cosmological model predicts $D_{\mathrm{L}}$, while what is actually observed is the flux. We can deduce the absolute luminosity only on the basis of some empirical methods. Similarly with galaxy number counts - the cosmological model predicts $m(z) n(z)$ where $m(z)$ is an average mass per source and $n(z)$ is number counts. The whole information about the galaxy evolution and their mergers is encoded in $m(z)$ - however in galaxy redshift surveys we observe only $n(z)$. In this paper we do not focus on the problem of observations and assume $D_{\mathrm{L}}$ and $m n$ as in the $\Lambda \mathrm{CDM}$.
} 


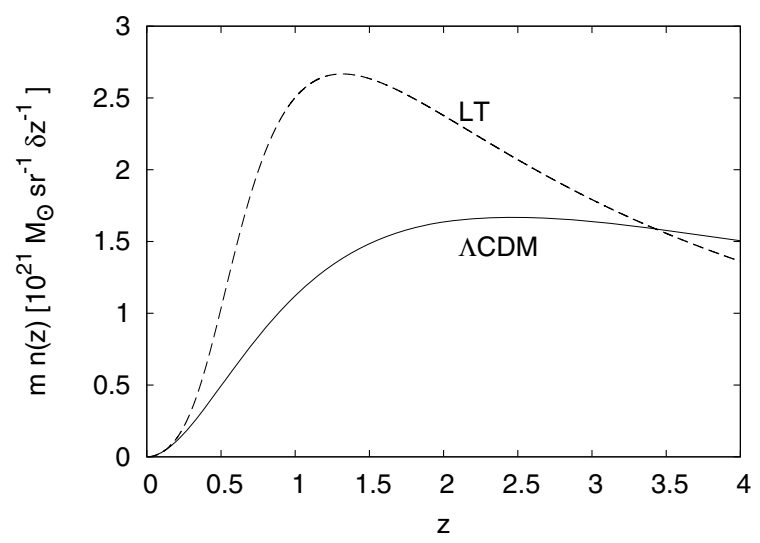

Fig. 1. The redshift-space mass-density in the $\Lambda \mathrm{CDM}$ model and in a giant void L-T model (with radius of $2.96 \mathrm{Gpc}$ and density contrast of 4.05). At the redshift around 1 one should observe twice as many objects as in the $\Lambda$ CDM model.

As is usual in the study of L-T models, we choose to use a comoving and synchronous coordinate system for the majority of this work. Such a coordinate system is uniquely defined by the flow lines of the fluid and allows the line-element to be written in a simple form. However, it is of course the case that quantities such as energy density profiles on space-like volumes are sensitive to the choice of hypersurface on which they are recorded. To illustrate this dependence, and the effect of considering other foliations, we also present our final results on a set of hypersurfaces in which each fluid element is the same distance from the initial singularity along the world-lines of the dust particles. Such a choice allows us to consider the energy density of different regions when they are at the same age, and becomes a comoving and synchronous coordinate system in the constant bang time models where giant voids have often been inferred.

In Sect. 2 we recall briefly the main properties of the L-T solution and give the equations to be integrated on the light cone. In Sects. 3.1 and 3.2 we describe both models and give the results of our numerical calculations. Section 4 is devoted to a discussion and a summary of our conclusions.

\section{The Lemaître-Tolman solution}

The L-T model is one of two classes of spherically symmetric solutions ${ }^{3}$ of Einstein's equations where the gravitational source is dust. In comoving and synchronous coordinates, its line element reads

$\mathrm{d} s^{2}=c^{2} \mathrm{~d} t^{2}-\frac{R, r^{2}}{1+2 E(r)} \mathrm{d} r^{2}-R^{2}(t, r)\left(\mathrm{d} \vartheta^{2}+\sin ^{2} \vartheta \mathrm{d} \varphi^{2}\right)$,

where $E(r)$ is an arbitrary function and $R, r=\partial R / \partial r$. With a vanishing cosmological constant, which is the case we consider here, $R(t, r)$ obeys the following first integral of one of the Einstein equations:

$\frac{1}{c^{2}} R,{ }^{2}=2 E+\frac{2 M}{R}$,

where $R,{ }_{t}=\partial R / \partial t$ and $M=M(r)$ is another arbitrary function of integration. $M(r)$ is the gravitational mass contained within the

${ }^{3}$ For the presentation of the other class see Plebański \& Krasiński (2006). It is called there the Datt-Ruban solution (Ruban 1968, 1969). It has interesting geometrical and physical properties, but so far has found no astrophysical application. comoving spherical shell at any given $r$, while $E(r)$ is the energy per unit mass of the particles on that shell. Moreover, $E$ determines the space curvature at each $r$-value. The mass density in energy units follows from the other nontrivial Einstein equation and is

$\kappa \rho c^{2}=\frac{2 M, r}{R^{2} R, r}, \quad$ where $\kappa=\frac{8 \pi G}{c^{4}}$.

The solutions of (2) can be written as

$R(t, r)=\frac{M(r)}{\chi(r)} \phi(t, r), \quad c\left(t-t_{\mathrm{B}}(r)\right)=\frac{M(r)}{(\chi(r))^{3 / 2}} \xi(t, r)$,

where

1. when $E<0$ (elliptic evolution):

$$
\begin{aligned}
\chi(r) & =-2 E(r), \\
\phi & =1-\cos \eta, \\
\xi & =\eta-\sin \eta,
\end{aligned}
$$

2. when $E=0$ (parabolic evolution):

$$
\begin{aligned}
\chi(r) & =1, \\
\phi & =\eta^{2} / 2, \\
\xi & =\eta^{3} / 6,
\end{aligned}
$$

3. and when $E>0$ (hyperbolic evolution):

$$
\begin{aligned}
\chi(r) & =2 E(r), \\
\phi & =\cosh \eta-1, \\
\xi & =\sinh \eta-\eta,
\end{aligned}
$$

where $\eta$ is a parameter (dependent on $t$ and $r$ ). The $t_{\mathrm{B}}(r)$ is another arbitrary function that appears as an integration "constant" and is interpreted as the bang time, i.e., the Big Bang is not simultaneous for all values of the $r$ coordinate.

Since all the formulae given so far are covariant under coordinate transformations of the form $\widetilde{r}=g(r)$, one of the functions $E(r), M(r)$ and $t_{\mathrm{B}}(r)$ can be fixed at will by the choice of $g$. Therefore, once this choice is done, a given L-T model is fully determined by two of these arbitrary functions.

However, as shown by Mustapha et al. (1998), and used as an illustration for application to the supernova data and the "cosmological constant problem" by Célérier (2000), a set of isotropic data corresponding to a given observable can constrain only one of the two free functions, and therefore the fitting of the supernova data, i.e. of the function $D_{\mathrm{L}}(z)$, with a given L-T solution, still leaves the other function free - and available for fitting to another set of data. Thus, we must also assume another set of initial conditions, e.g. the redshift-space mass-density $m(z) n(z)$ or the expansion rate $H(z)$. We will take these functions to be identical to the corresponding functions in the $\Lambda$ CDM model, assuming they reflect the observational data. By this, we want to show that there is no antagonism between the inhomogeneous cosmology and the $\Lambda \mathrm{CDM}$ model, and that the first can predict the same results as the second even if $\Lambda=0$. However, whenever possible we present the real data to show that there is still much room within observational errors for different profiles.

Using the reciprocity theorem (Etherington 1933; Ellis 1971) the luminosity distance can be converted to the angular diameter distance

$D_{\mathrm{A}}=R=\frac{D_{\mathrm{L}}}{(1+z)^{2}}$. 
For a ray issued from a radiating source and proceeding towards the central observer on a radial null geodesic the following equation holds

$\frac{c \mathrm{~d} t(r)}{\mathrm{d} r}=-\frac{R, r(t(r), r)}{\sqrt{1+2 E(r)}}$,

and the equation for the redshift reads (Bondi 1947; Plebański \& Krasiński 2006):

$\frac{1}{1+z(r)} \frac{\mathrm{d} z(r)}{\mathrm{d} r}=\frac{1}{c} \frac{R, \mathrm{tr}(t(r), r)}{\sqrt{1+2 E(r)}}$.

For later reference we will need also the following equation, which follows easily from (9) and (10):

$\frac{1}{1+z(t)} \frac{\mathrm{d} z(t)}{\mathrm{d} t}=-\frac{R, \mathrm{tr}(t, r(t))}{R, r(t, r(t))}$.

\section{The L-T model with no central void}

Our aim is now to show we can design an L-T model with no central void able to reproduce the angular diameter distanceredshift relation as inferred from the SN Ia data, smoothed out as in the framework of a $\Lambda \mathrm{CDM}$ model. For this purpose, we propose to use the following additional conditions to specify the arbitrary functions $M(r), E(r)$ and $t_{\mathrm{B}}(r)$.

\subsection{The model defined by $D_{A}(z)$ and $m(z) n(z)$}

\subsubsection{The MHE procedure}

The algorithm used to find the L-T model reproducing the $D_{\mathrm{A}}(z)$ and $n(z)$ data was first developed by Mustapha et al. (1997). Let us recall its major steps and equations.

The radial coordinate $r$ is chosen so that, on the past light cone of $(t, r)=\left(t_{0}, 0\right)$

$$
\frac{\widehat{R_{r}}}{\sqrt{1+2 E}}=1 \text {. }
$$

(Note: this choice of $r$ is possible only on a single light cone. In the following, we always refer to the light cone of $(t, r)=\left(t_{0}, 0\right)$.) This choice of coordinates simplifies the null geodesic equation:

$\widehat{c t}(r)=c t_{0}-r$,

where we denote quantities on this null cone by a hat. Furthermore, (3) now becomes

$\left(\kappa c^{2} / 2\right) \widehat{\rho} \widehat{R}^{2}=\frac{M, r}{\sqrt{1+2 E}}$.

The total derivative of the areal radius $R$ gives

$\frac{\mathrm{d} \widehat{R}}{\mathrm{~d} r}=\widehat{R, r}+\widehat{R, t} \frac{\mathrm{d} \widehat{d}}{\mathrm{~d} r}$.

Using (2), (12) and (13), the above equation can be written as

$\frac{\mathrm{d} \widehat{R}}{\mathrm{~d} r}-\sqrt{1+2 E}=-\frac{1}{c} \widehat{R, t}=\mp \sqrt{\frac{2 M}{\widehat{R}}+2 E}$.

This can be solved for $E(r)$ :

$1+2 E=\left\{\frac{1}{2}\left[\left(\frac{\mathrm{d} \widehat{R}}{\mathrm{~d} r}\right)^{2}+1\right]-\frac{M}{\widehat{R}}\right\}^{2} /\left(\frac{\mathrm{d} \widehat{R}}{\mathrm{~d} r}\right)^{2}$.
Using (14) the above becomes

$\frac{\mathrm{d} M}{\mathrm{~d} r}+\left(\frac{\kappa c^{2} \widehat{\rho} \widehat{R}}{2 \mathrm{~d} \widehat{R} / \mathrm{d} r}\right) M=\left(\frac{\kappa c^{2} \widehat{\rho} \widehat{R}^{2}}{4 \mathrm{~d} \widehat{R} / \mathrm{d} r}\right)\left[\left(\frac{\mathrm{d} \widehat{R}}{\mathrm{~d} r}\right)^{2}+1\right]$.

Matter density can be expressed in terms of $n(z)$ - the observed number density of sources in the redshift space per steradian per unit redshift interval. Thus, the number of sources observed in a given redshift interval and solid angle $\mathrm{d} \Omega$ is $n \mathrm{~d} \Omega \mathrm{d} z$ and the total rest mass between $z$ and $z+\mathrm{d} z$ is

$\mathcal{M}=4 \pi \widehat{m n} \mathrm{~d} z$,

where $\widehat{m}(z)$ is the average mass per source. On the other hand the total rest mass between $r$ and $r+\mathrm{d} r$ is

$\mathcal{M}=\widehat{\rho} \mathrm{d}^{3} V=\widehat{\rho} \frac{4 \pi \widehat{R}^{2} \widehat{R, r}}{\sqrt{1+2 E}} \mathrm{~d} r$

where $\widehat{\mathrm{d}^{3} V}$ is the proper volume on a constant time slice, evaluated on the null cone. Hence by (19), (20) and (12)

$\widehat{R}^{2} \widehat{\rho}=\widehat{m n} \frac{\mathrm{d} z}{\mathrm{~d} r}$.

Finally, to find $r(z)$, the r.h.s. of (10) must be expressed in terms of $\widehat{R}$ and $\widehat{n}(z)$. Differentiating (2) with respect to $r$ and substituting the result in the r.h.s. of (10), we obtain

$\frac{\widehat{R, \text { tr }}}{\sqrt{1+2 E}}=\frac{c^{2}}{\widehat{R, t}}\left[\frac{M, r}{\widehat{R} \sqrt{1+2 E}}-\frac{M}{\widehat{R}^{2}}+(\sqrt{1+2 E})_{, r}\right]$.

The derivative of $\sqrt{1+2 E}$ follows from (17). Then, replacing $M, r$ by (14) and using (16), the above equation can be written as

$\frac{1}{c} \frac{\widehat{R, \text { tr }}}{\sqrt{1+2 E}}=-\left(\frac{1}{2} \kappa c^{2} \widehat{\rho} \widehat{R}+\frac{\mathrm{d}^{2} \widehat{R}}{\mathrm{~d} r^{2}}\right) /\left(\frac{\mathrm{d} \widehat{R}}{\mathrm{~d} r}\right)$.

Now, from (10):

$\frac{\mathrm{d} \widehat{R}}{\mathrm{~d} r} \frac{\mathrm{d} z}{\mathrm{~d} r}+\frac{\mathrm{d}^{2} \widehat{R}}{\mathrm{~d} r^{2}}(1+z)=-\frac{1}{2} \kappa c^{2} \widehat{\rho} \widehat{R}(1+z)$.

Applying

$\frac{\mathrm{d} \widehat{R}}{\mathrm{~d} r}=\frac{\mathrm{d} \widehat{R}}{\mathrm{~d} z} \frac{\mathrm{d} z}{\mathrm{~d} r}, \quad \frac{\mathrm{d}^{2} \widehat{R}}{\mathrm{~d} r^{2}}=\frac{\mathrm{d} \widehat{R}}{\mathrm{~d} z} \frac{\mathrm{d}^{2} z}{\mathrm{~d} r^{2}}+\frac{\mathrm{d}^{2} \widehat{R}}{\mathrm{~d} z^{2}}\left(\frac{\mathrm{d} z}{\mathrm{~d} r}\right)^{2}$,

and integrating with respect to $r$, yields

$$
\begin{aligned}
\int_{0}^{z} \frac{\mathrm{d}}{\mathrm{d} r} & {\left[\frac{\mathrm{d} \bar{z}}{\mathrm{~d} r} \frac{\mathrm{d} \widehat{R}}{\mathrm{~d} \bar{z}}(1+\bar{z})\right] \mathrm{d} r } \\
& =-\int_{0}^{z} \frac{1}{2} \kappa c^{2} \widehat{\rho}(\bar{z}) \widehat{R}(\bar{z})(1+\bar{z}) \frac{\mathrm{d} r}{\mathrm{~d} \bar{z}} \mathrm{~d} \bar{z} .
\end{aligned}
$$

Using the origin conditions $\left.[(\mathrm{d} z / \mathrm{d} r)(\mathrm{d} \widehat{R} / \mathrm{d} z)]_{0}=[\mathrm{d} \widehat{R} / \mathrm{d} r)\right]_{0}=1$, $z(0)=0$ and $(21)$ the above can be rearranged to obtain

$$
\begin{aligned}
\frac{\mathrm{d} z}{\mathrm{~d} r}= & {\left[\frac{\mathrm{d} \widehat{R}}{\mathrm{~d} z}(1+z)\right]^{-1} } \\
& \times\left\{1-\frac{1}{2} \kappa c^{2} \int_{0}^{z} \frac{\widehat{m}(\bar{z}) n(\bar{z})}{\widehat{R}(\bar{z})}(1+\bar{z}) \mathrm{d} \bar{z}\right\}
\end{aligned}
$$

Finally:

$$
\begin{aligned}
r(z)= & \int_{0}^{z}\left[\frac{\mathrm{d} \widehat{R}}{\mathrm{~d} \tilde{z}}(1+\tilde{z})\right] \\
& \times\left\{1-\frac{1}{2} \kappa c^{2} \int_{0}^{\tilde{z}} \frac{\widehat{m}(\bar{z}) n(\bar{z})}{\widehat{R}(\bar{z})}(1+\bar{z}) \mathrm{d} \bar{z}\right\}^{-1} \mathrm{~d} \tilde{z} .
\end{aligned}
$$


M. N. Célérier et al.: Explaining away dark energy without a (giant) void

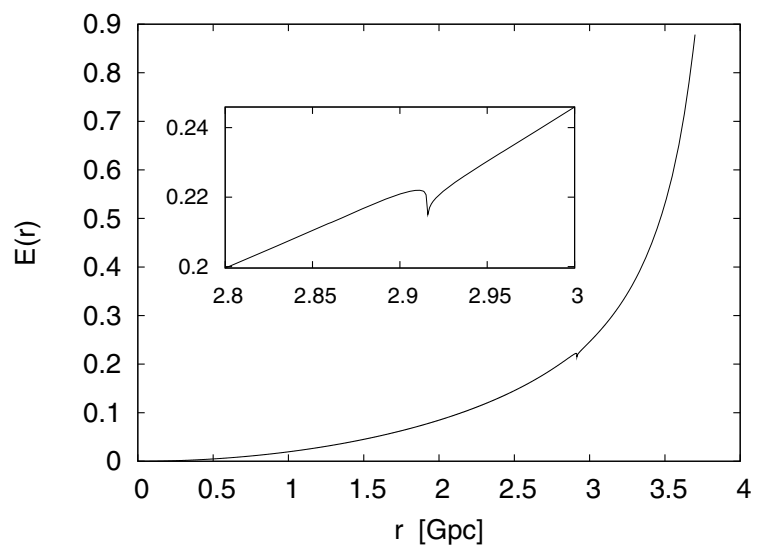

Fig. 2. The function $E(r)$ of the L-T model defined by the $\left(D_{\mathrm{A}}, m(z) n(z)\right)$ set of data (see Sect. 3.1.1, for details). There is a problem with numerical integration around $r=2.9 \mathrm{Gpc}$. The problem comes from (17) where the term $(1-2 M / R) /(\mathrm{d} R / \mathrm{d} z)$ becomes $0 / 0$ at the apparent horizon.

\subsubsection{The algorithm}

In order to specify the model, we proceed in the following way:

1. The model is defined by two functions on the past null cone: the angular diameter distance, $D_{\mathrm{A}}(z)$, and the mass density in redshift space, $m(z) n(z)$. We assume that these functions are the same as in the $\Lambda \mathrm{CDM}$ model:

$$
\begin{aligned}
& D_{\mathrm{A}}(z)=\frac{1}{1+z} \frac{c}{H_{0}} \int_{0}^{z} \frac{\mathrm{d} z^{\prime}}{\sqrt{\Omega_{\mathrm{m}}\left(1+z^{\prime}\right)^{3}+1-\Omega_{\mathrm{m}}}}, \\
& m(z) n(z)=\Omega_{\mathrm{m}} \frac{3 H_{0}^{2}}{8 \pi G}(1+z)^{3} D_{\mathrm{A}}^{2} \frac{\mathrm{d} r}{\mathrm{~d} z},
\end{aligned}
$$

where $\Omega_{\mathrm{m}}=(8 \pi G) /\left(3 H_{0}^{2}\right) \rho_{0}$.

2. Using the MHE algorithm we find $r(z)$ by solving (27).

3. We numerically invert this relation to find $z(r)$ and solve (18) to find $M(r)$.

4. The function $E(r)$ is found by solving (17).

5. Once $E$ and $M$ are known, we find $\eta$ and then $t_{\mathrm{B}}$, by solving the appropriate relations (4)-(7).

6. Since in (17) the term $(1-2 M / R) /(\mathrm{d} R / \mathrm{d} z)$ becomes $0 / 0$ at the apparent horizon, the computer produces inaccurate results in the vicinity. To overcome this we apply the procedure described in Sect. 3.1.4.

\subsubsection{The results}

The algorithm described in the previous section allows us to find an L-T model from a given $\left(D_{\mathrm{A}}(z), m(z) n(z)\right)$ set of data.

The free functions of the L-T model, $E, t_{\mathrm{B}}$, and $M$ are shown in Figs. 2, 3, and 4 respectively.

As can be seen, there is a problem with numerical integration for $E$ and $t_{\mathrm{B}}$ around $r=2.9 \mathrm{Gpc}$. The problem is related to $(17)$ where the term $(1-2 M / R) /(\mathrm{d} R / \mathrm{d} z)$ becomes $0 / 0$ at the apparent horizon. Because of this, the computer produces inaccurate results in the vicinity. One solution to this problem was proposed by Lu \& Hellaby (2007) who performed series expansions of $R(z), n(z), \mathrm{d} r / \mathrm{d} z, M(z)$ and $E(z)$ around the apparent horizon. However, this method leads either to jumps in one of these functions, say $E(z)$, or to lower accuracy of the algorithm (Lu \& Hellaby 2007). Therefore, we propose a different, much simpler approach. Namely, we fit polynomials to $E(r)$ and $M(r)$ and then we recalculate the area distance and the redshift-space

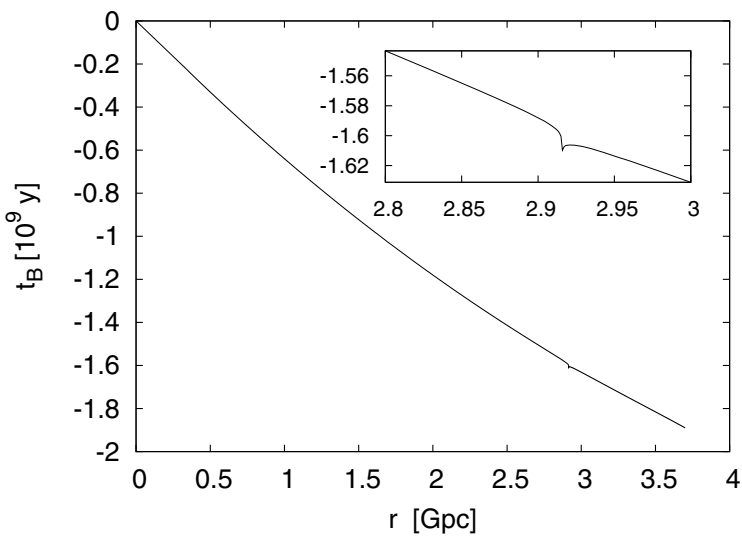

Fig. 3. The function $t_{\mathrm{B}}(r)$ of the L-T model defined by the $\left(D_{\mathrm{A}}, m(z) n(z)\right)$ set of data (see Sect. 3.1.1, for details). There is a cusp around $r=$ $2.9 \mathrm{Gpc}$. The cusp follows from an unstable behaviour of $E(r)$ around $r=2.9 \mathrm{Gpc}$.

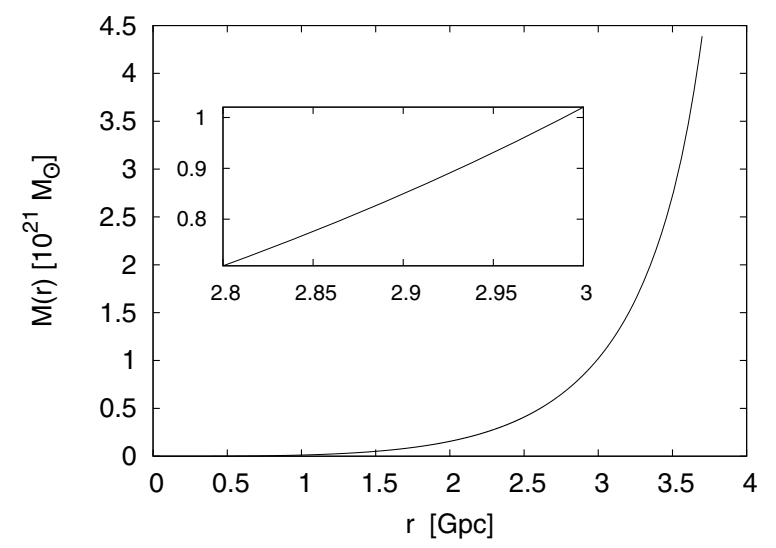

Fig. 4. The function $M(r)$ of the L-T model defined by the $\left(D_{\mathrm{A}}, m(z) n(z)\right)$ set of data (see Sect. 3.1.1, for details). In this case there is no problem around $r=2.9 \mathrm{Gpc}$.

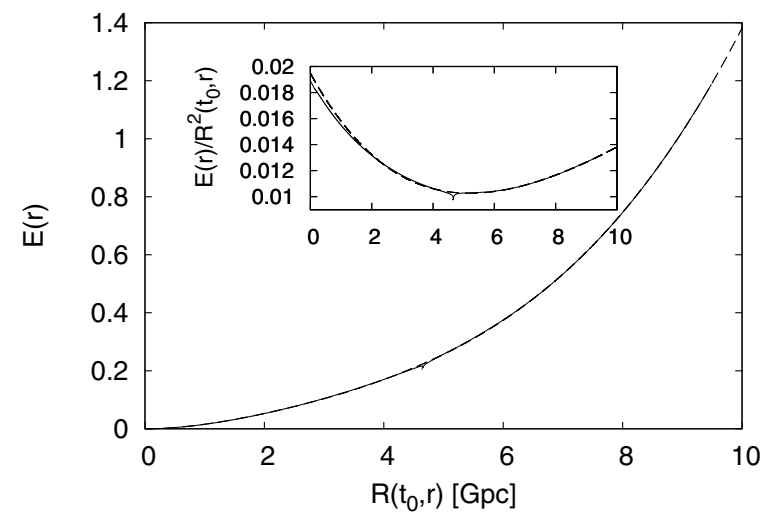

Fig. 5. The function $E$ as a function of the current areal radius for the $\left(D_{\mathrm{A}}, m(z) n(z)\right)$ set of data - the comparison of results obtained for $E$ as given by (17) (solid line) and for the approximation to $E$ as given by (31) (dashed line). See Sect. 3.1.4 for details. The inset presents $E / R^{2}$, the quantity that is constant in the FLRW limit.

mass-density as functions of redshift to check the accuracy of our approximations. As we will see, this method leads to results that from the observational point of view (every observation is accompanied with an error) are indistinguishable from those of the $\Lambda \mathrm{CDM}$ model. 


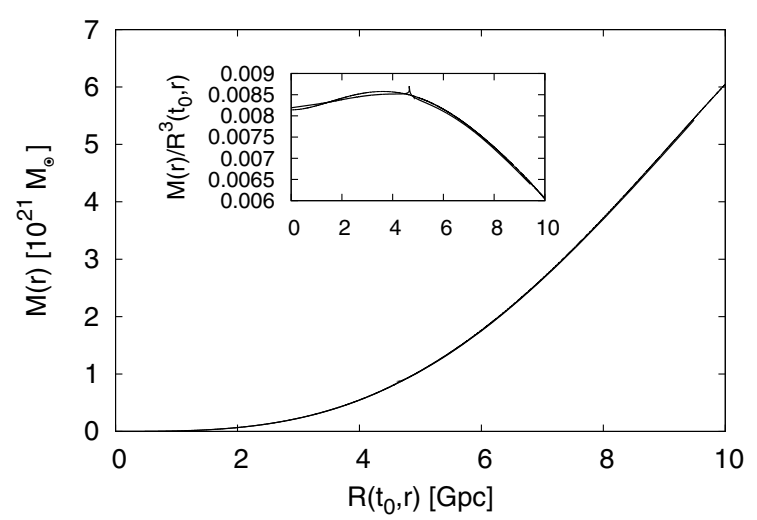

Fig. 6. The function $M$ as a function of the current areal radius for the $\left(D_{\mathrm{A}}, m(z) n(z)\right)$ set of data - the comparison of results obtained for $M$ as given by (18) (solid line) and for the approximation to $M$ as given by (30) (dashed line). See Sect. 3.1.4 for details. The inset presents $M / R^{3}$, the quantity that is constant in the FLRW limit.

\subsubsection{Dealing with the apparent horizon}

To overcome the numerical problem of indeterminacy of $E(r)$ at the apparent horizon, we fit polynomials to the obtained $M(r)$ and $E(r)$. The most obvious choice would be polynomials in the variable $r$. However, in numerical experiments we noticed that much better results are obtained when we approximate $M$ and $E$ by polynomials in $R\left(t_{0}, r\right)$, where $t_{0}$ is the present instant. The explicit forms of the fitted functions are

$M\left(R\left(t_{0}, r\right)\right)=\sum_{n=3}^{n=8} M_{n} \ell^{n}$

where $\ell=R\left(t_{0}, r\right) / 1 \mathrm{Gpc},\left(M_{3}, M_{4}, M_{5}, M_{6}, M_{7}, M_{8}\right)=$ $\left(8.142244 \times 10^{-3} \mathrm{kpc}, 0 \mathrm{kpc}, 1.32458 \times 10^{-4} \mathrm{kpc},-3.79 \times\right.$ $\left.10^{-5} \mathrm{kpc}, 3.23834 \times 10^{-6} \mathrm{kpc},-9.8233 \times 10^{-8} \mathrm{kpc}\right)$, and

$E\left(R\left(t_{0}, r\right)\right)=\sum_{n=2}^{n=6} E_{n} \ell^{n}$,

where $\left(E_{2}, E_{3}, E_{4}, E_{5}, E_{6}\right)=\left(1.9475 \times 10^{-2}, 4.28698 \times 10^{-3}\right.$, $\left.6.50383 \times 10^{-4},-3.66095 \times 10^{-5}, 8.78679 \times 10^{-7}\right)$.

The profiles of these functions together with those numerically derived are presented in Figs. 5 and 6. We then use these functions as initial conditions and solve the null geodesic equations. First, we invert (10) and (11) to get the equations for $\mathrm{d} t / \mathrm{d} z$ and $\mathrm{d} r / \mathrm{d} z$, to derive the pair $(t, r)$ for a given redshift; simultaneously we solve (2) to get $\widehat{R}, \widehat{R, r}$, and $\widehat{R, \text { tr }}$.

The results are shown in Figs. 7, 8. The angular diameter distance is recovered very accurately, while the redshift-space mass-density less so, but still up to $z=4$ it does not differ by more than $6 \%$ from the redshift-space mass-density in the $\Lambda C D M$ model - which is far less than the expected observational uncertainty. In addition we calculate the prediction for $H(z)$, and we compare it to the estimations of the expansion rate by Simon et al. (2005). Since these are based on the observed age of the oldest stars, and $H(z)$ follows from $\mathrm{d} t / \mathrm{d} z$, thus, as seen from (11), $H(z)=\widehat{R, \text { tr }} / \widehat{R, r}$. The results are presented in Fig. 9. As seen, the L-T model does not deviate from the $\Lambda$ CDM model by more than $5 \%$. These differences in $m(z) n(z)$ and $H(z)$ are caused by two factors: a) the Eqs. (30) and (31) are just approximations; b) numerical errors in the vicinity of the apparent horizon bias the solution of (17) for $r \gtrsim r_{\mathrm{AH}}$ (where $r_{\mathrm{AH}}$ is the position of the

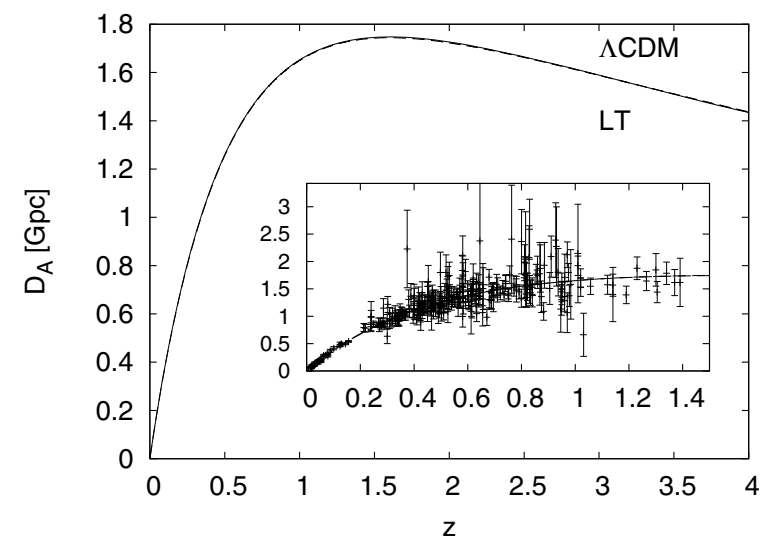

Fig. 7. The angular diameter distance as a function of redshift; comparison of the results for the $\Lambda \mathrm{CDM}$ and L-T models. The inset presents the estimations of $D_{\mathrm{A}}$ based on the type-Ia supernova measurements taken from the Union data set (Kowalski et al. 2008). See Sect. 3.1.4.

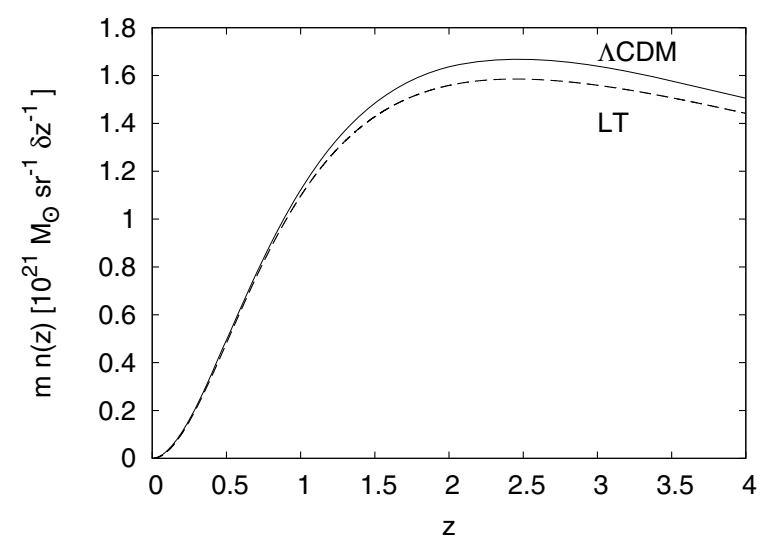

Fig. 8. The redshift-space mass-density as a function of redshift. The difference between the LT and $\Lambda$ CDM models is less than $6 \%$. See Sect. 3.1.4.

apparent horizon). In principle, however, it is possible to construct the $\Lambda=0 \mathrm{~L}-\mathrm{T}$ model that matches the $\Lambda \mathrm{CDM}$ model. Finally, as seen from Fig. 10, the current density profile does not exhibit a giant void shape. Instead, it suggests that the universe smoothed out around us with respect to directions is overdense in our vicinity up to Gpc-scales. As a consequence of our numerical procedure the value of density at the centre at the present time $t_{0}$ is the same as the present density in the $\Lambda$ CDM model.

\subsection{The model defined by $D_{\mathrm{A}}(z)$ and $H(z)$}

\subsubsection{The algorithm}

The algorithm used to find the L-T model consists of the following steps:

1. The model is defined by two functions on the past null cone: the angular diameter distance $D_{\mathrm{A}}(z)$ and the Hubble function $H(z)$. We assume that these functions are the same as in the $\Lambda \mathrm{CDM}$ model $-D_{\mathrm{A}}(z)$ is given by (28) and

$$
H(z)=H_{0} \sqrt{\Omega_{\mathrm{m}}(1+z)^{3}+1-\Omega_{\mathrm{m}}} .
$$


M. N. Célérier et al.: Explaining away dark energy without a (giant) void

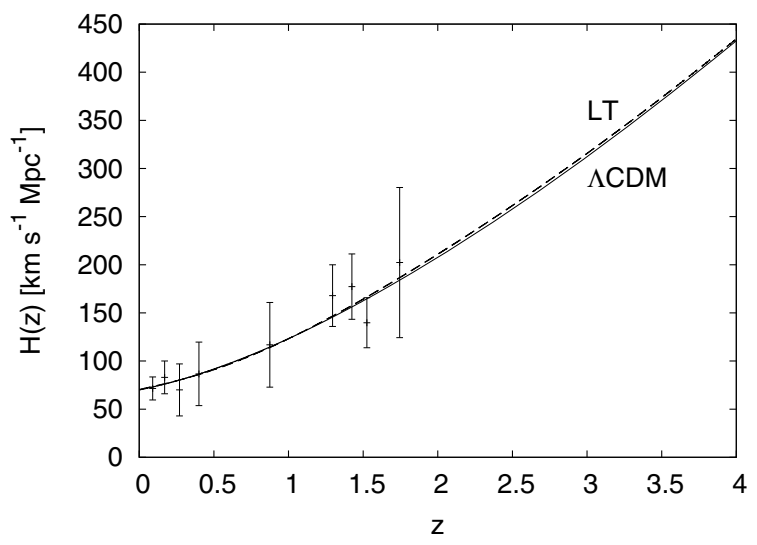

Fig. 9. The function $H(z)$. See Sect. 3.1.4 for details. For comparison the measurements of $H(z)$ (Simon et al. 2005) are also shown. The difference between the LT and $\Lambda$ CDM models is less than $5 \%$.

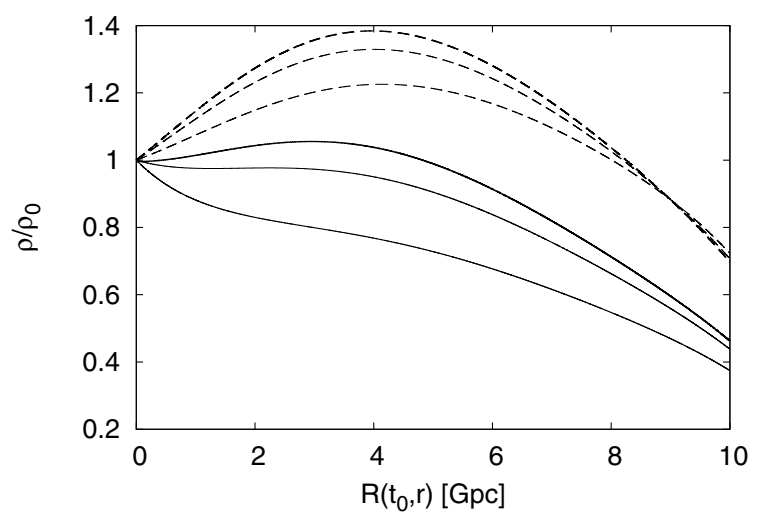

Fig. 10. The ratio $\rho / \rho_{0}$ of density to density at the origin. Solid lines present density profiles at constant $t$ (from top to bottom: $t=$ now, $t=$ now $\left.-2 \times 10^{9} \mathrm{y}, t=n o w-5 \times 10^{9} \mathrm{y}\right)$. Dashed lines present density profiles at the hypersurface of constant age of the Universe (from top to bottom: $\tau=$ now, $\tau=$ now $-2 \times 10^{9} \mathrm{y}, \tau=$ now $-5 \times 10^{9} \mathrm{y}$ ). For each curve, the $\rho_{0}$ is taken at the value of $t$ or, respectively, $\tau$ that identifies the curve. Note: all the graphs, for both foliations, use a comoving radial coordinate, $r:=$ $R\left(t_{0}, r\right)$, which means that points with the same horizontal coordinate in the graph correspond to the same matter particle at all times. Only for the uppermost solid graph is $R\left(t_{0}, r\right)$ equal to the actual area-distance from the centre.

2. We choose $r$ so that (12) is satisfied on the past light cone of the present-day observer. Then using $H(z)=\widehat{R, t r} / \widehat{R, r},(10)$ becomes

$\frac{\mathrm{d} r}{\mathrm{~d} z}=\frac{1}{1+z} \frac{c}{H(z)}$,

which for (32) can be integrated to

$$
\begin{aligned}
r= & \frac{2 c}{3 H_{0}} \frac{1}{\sqrt{1-\Omega_{\mathrm{m}}}}\left[\operatorname{arsinh}\left(\sqrt{\frac{1-\Omega_{\mathrm{m}}}{\Omega_{\mathrm{m}}}}\right)\right. \\
& \left.-\operatorname{arsinh}\left(\sqrt{\frac{1-\Omega_{\mathrm{m}}}{\Omega_{\mathrm{m}}(1+z)^{3}}}\right)\right] .
\end{aligned}
$$

3. Using (23) we find $\rho(z)$ and solve (21) for $n(z)$.

4. We solve (18) to find $M(r)$.

5. The function $E(r)$ is found by solving (17).

6. Once $E$ and $M$ are known we find $\eta$ and then $t_{\mathrm{B}}$ by solving the appropriate relations (4)-(7).

7. As before, because of the $0 / 0$ term in (17) at the apparent horizon, we employ the procedure described in Sect. 3.2.3.

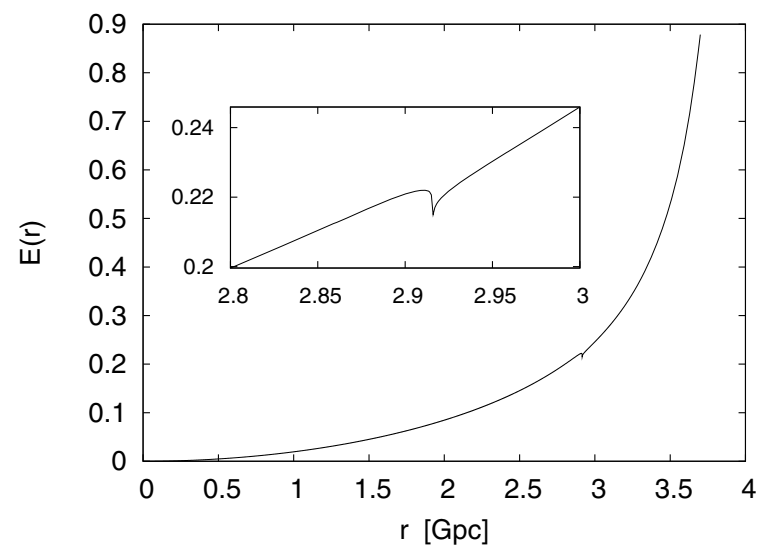

Fig. 11. The function $E(r)$ of the L-T model defined by the $\left(D_{\mathrm{A}}, H(z)\right)$ set of data (see Sect. 3.2.1, for details). Around $r=2.9 \mathrm{Gpc}$ there is a problem with the numerical algorithm.

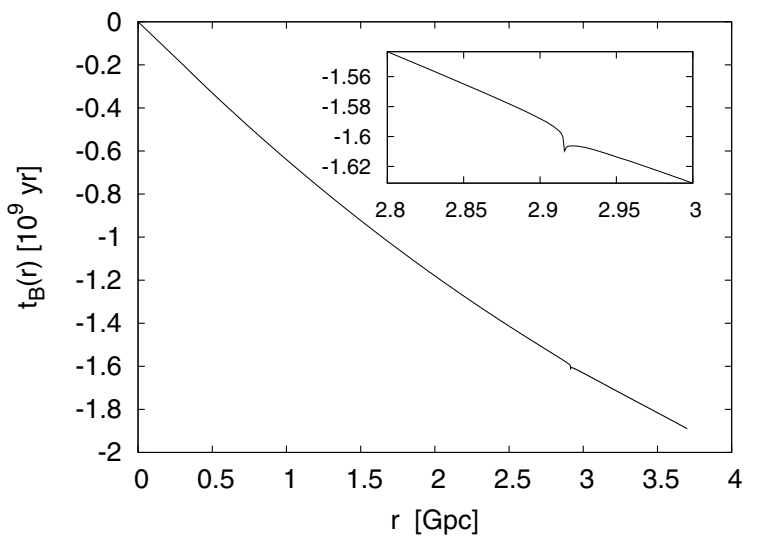

Fig. 12. The function $t_{\mathrm{B}}(r)$ of the L-T model defined by the $\left(D_{\mathrm{A}}, H(z)\right)$ set of data (see Sect. 3.2.1, for details).

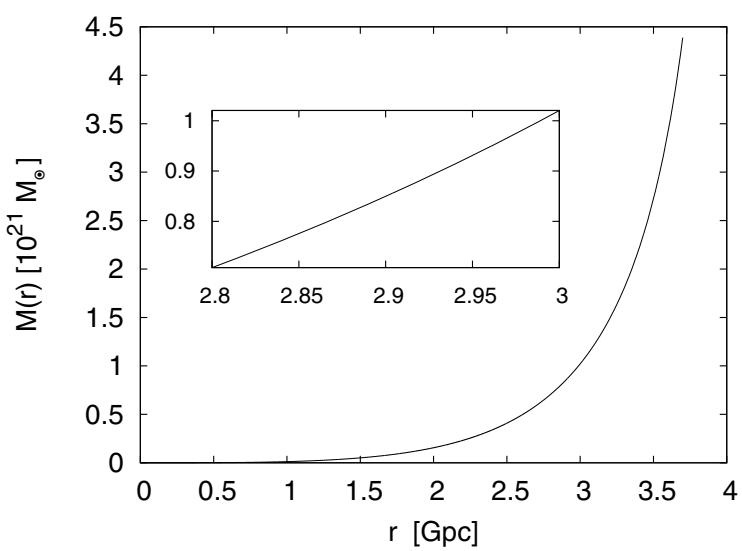

Fig. 13. The function $M(r)$ of the L-T model defined by the $\left(D_{\mathrm{A}}, H(z)\right)$ set of data (see Sect. 3.2.1, for details). In this case there is no problem around $r=2.9 \mathrm{Gpc}$.

\subsubsection{The results}

The results for $E, t_{\mathrm{B}}$ and $M$ are given in Figs. 11-13. As in Sect. 3.1.3, the functions $E(r)$ and $t_{\mathrm{B}}(r)$ evaluated by this algorithm behave unnaturally close to the apparent horizon, see Figs. 11 and 12. As before, this is caused by the fact that in (17) one has to deal with $0 / 0$. We overcome this problem by once again fitting polynomials to these functions. 


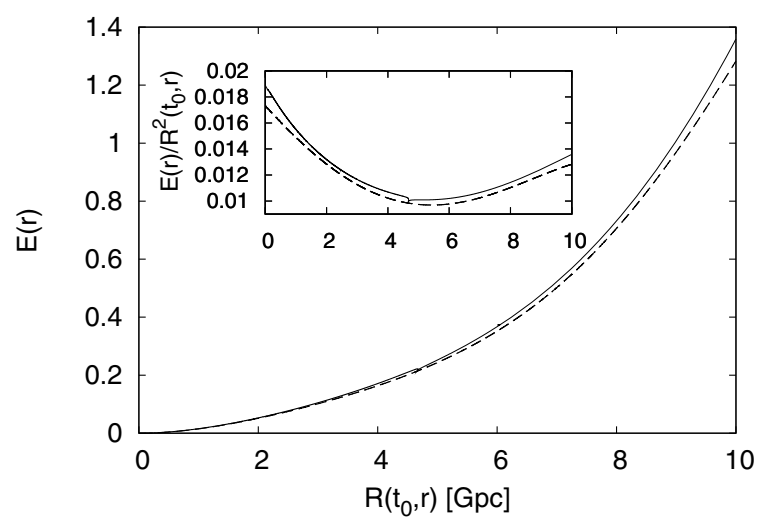

Fig. 14. The function $E$ as a function of the current areal radius for the $\left(D_{\mathrm{A}}, H(z)\right)$ set of data - the comparison of results obtained for $E$ as given by (17) (solid line) and for the approximation to $E$ as given by (36) (dashed line). See Sect. 3.2.3 for details. The inset presents $E / R^{2}$, the quantity that is constant in the FLRW limit.

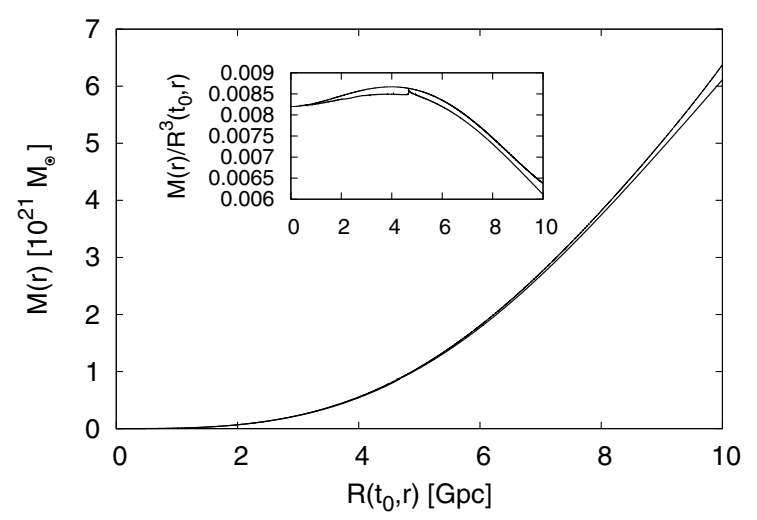

Fig. 15. The function $M$ as a function of the current areal radius for the $\left(D_{\mathrm{A}}, H(z)\right)$ set of data - the comparison of results obtained for $M$ as given by (18) (solid line) and for the approximation to $M$ as given by (35) (dashed line). See Sect. 3.2.3 for details. The inset presents $M / R^{3}$, the quantity that is constant in the FLRW limit.

\subsubsection{Dealing with the apparent horizon}

The explicit forms of the fitted functions are:

$M\left(R\left(t_{0}, r\right)\right)=\sum_{n=3}^{n=7} M_{n} \ell^{n}$,

where $\left(M_{3}, M_{4}, M_{5}, M_{6}, M_{7}\right)=\left(8.2 \times 10^{3} \mathrm{kpc},-1.1948 \mathrm{kpc}\right.$, $\left.1.07521 \times 10^{2} \mathrm{kpc},-24.1385 \mathrm{kpc}, 1.15743 \mathrm{kpc}\right)$, and

$E\left(R\left(t_{0}, r\right)\right)=\sum_{n=2}^{n=6} E_{n} \ell^{n}$,

where $\left(E_{2}, E_{3}, E_{4}, E_{5}, E_{6}\right)=\left(1.7324 \times 10^{-2},-2.5725 \times 10^{-3}\right.$, $\left.1.14925 \times 10^{-4}, 2.87776 \times 10^{-5},-1.90389 \times 10^{-6}\right)$.

The profiles of these functions together with the numerically derived ones are shown in Figs. 14 and 15. We then use these functions as initial conditions and solve the null geodesic equations. The results are presented in Figs. 16 and 17. Both the angular diameter distance and the expansion rate as functions of the redshift are recovered very accurately. From the observational perspective these two models are indistinguishable. In addition we present the $m(z) n(z)$ plot. As seen from Fig. 18, it also gives quite an accurate fit, with a deviation from the $\Lambda$ CDM model

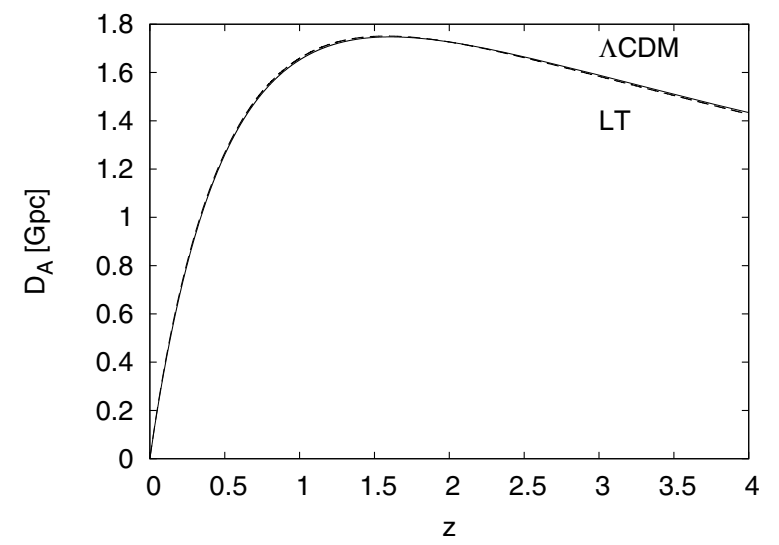

Fig. 16. The angular diameter distance as a function of $z$. See Sect. 3.2.3 for details.

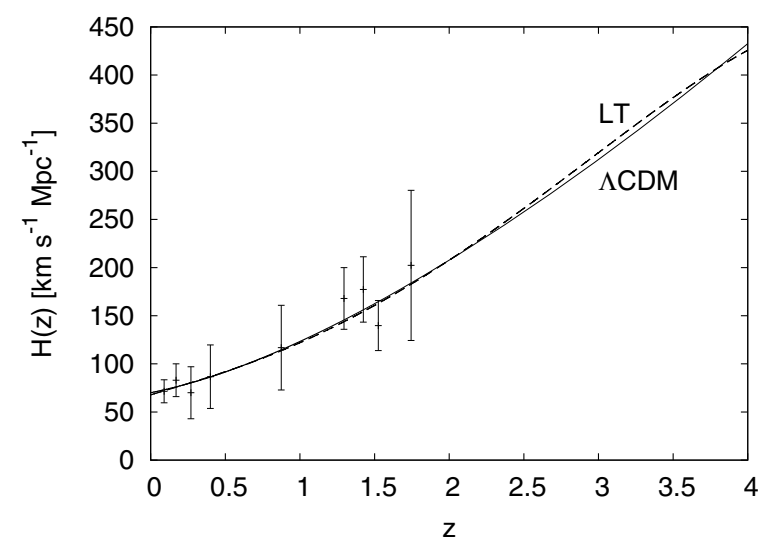

Fig. 17. The function $H(z)$. See Sect. 3.2.3 for details. For comparison the measurements of $H(z)$ (Simon et al. 2005) are also shown.

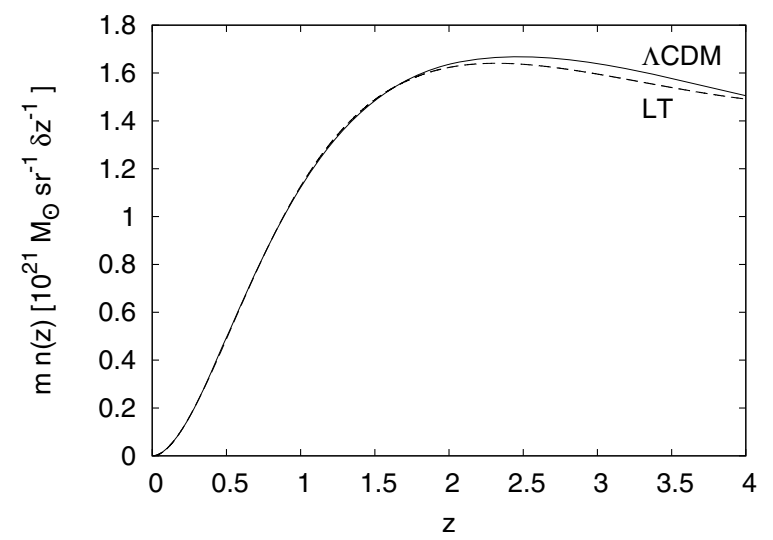

Fig. 18. The redshift-space mass-density as a function of redshift for the LT model considered in Sect. 3.2.3, and in the $\Lambda$ CDM model. The difference between the two models is less than $2.5 \%$. Compare Fig. 1; the consistency achieved here is much better than in a giant void model.

being less than $2.5 \%$. Finally, as seen from Fig. 19, the current density profile has a similar shape as in Sect. 3.1.4, and this is not a giant void.

\section{Discussion and conclusion}

Contrary to what is commonly claimed, L-T models with a giant void do not reproduce the main features of the $\Lambda$ CDM model. These types of models just fit cosmological observations, with a priori constraints imposed on the L-T models. Indeed, we have 
M. N. Célérier et al.: Explaining away dark energy without a (giant) void

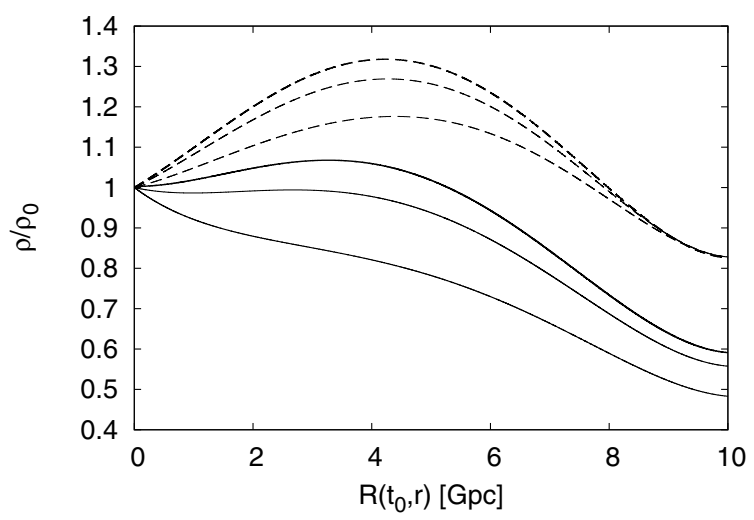

Fig. 19. The ratio $\rho / \rho_{0}$ of density to density at the origin. Solid lines present density profiles at constant $t$ (from top to bottom: $t=n o w$, $t=$ now $-2 \times 10^{9} \mathrm{y}, t=$ now $-5 \times 10^{9} \mathrm{y}$ ). Dashed lines present density profiles at the hypersurfaces of constant age of the Universe (from top to bottom: $\tau=$ now, $\tau=$ now $-2 \times 10^{9} \mathrm{y}, \tau=$ now $-5 \times 10^{9} \mathrm{y}$ ). For each curve, the $\rho_{0}$ is taken at the value of $t$ or, respectively, $\tau$ that identifies the curve. The remark about the horizontal coordinate in Fig. 10 applies also here.

found the L-T models that mimic some of the observational features of the $\Lambda$ CDM model and exhibit no giant void, but rather a giant hump.

It is clear from the energy density profiles shown in Figs. 10 and 19 that the L-T models we have reconstructed have a large overdensity, when viewed over large enough scales. However, as we have made clear throughout, these profiles are the result of reproducing observables that match $\Lambda \mathrm{CDM}$ predictions, and not from fitting to any real data. Luminosity distances from supernovae observations, and number counts from galaxy surveys do not currently extend much beyond $z \sim 1.5$ and $z \sim 0.5$, respectively. As such, using real observables, it would only currently be possible to perform a reconstruction of a limited part of the full structure we have found here, and even then only up to the degree allowed by the errors associated with these quantities. If one were to attempt such a reconstruction, it appears from Figs. 10 and 19 that one may, in fact, reconstruct a local energy density profile that is an increasing function of $r$, and not decreasing, due to the limited extent of these observations in $z$ (this is particularly true in the equal age foliation). Our interpretation of a giant hump should therefore be understood as corresponding to an extrapolation of observable quantities under the expectation that they will follow $\Lambda \mathrm{CDM}$, rather than being directly implied by any currently known observations themselves.

Recently, some astrophysicists have begun to take seriously the cosmological implications of the L-T model. This model, although still simple ${ }^{4}$, is quite powerful and exhibits some features of general relativistic dynamics, like arbitrary functions in the initial data.

As we said earlier in this paper, the belief that an L-T model fitted to supernova Ia observations necessarily implies the

\footnotetext{
${ }^{4}$ From the computational point of view, the difference between the Friedmann and L-T models is quite trivial. The Eq. (2) that governs the evolution of the L-T model is exactly the same as in the Friedmann model; it is still an ordinary differential equation in the time-variable $t$. The only difference is that the function $R(t)$ obeying (2) depends on one more variable, the radial coordinate $r$. This $r$ enters only as a parameter, and then automatically all the integration "constants" that appear while solving (2) are no longer constant, but are functions of $r$. Sophistication comes at the level of interpreting the solutions - however, this is no longer mathematics, but astrophysics.
}

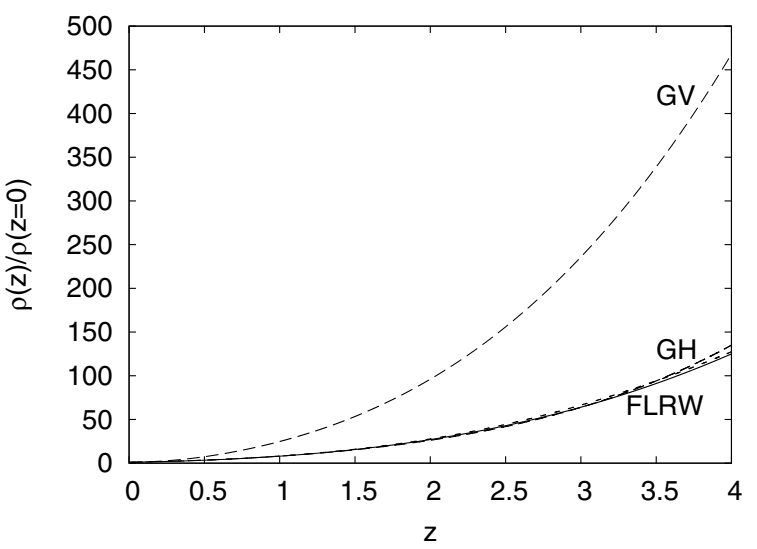

Fig. 20. The density distribution as a function of redshift for the giant void (GV) L-T model, for the models of Sect. 3.1.4 and 3.2.3 (GH) and for the FLRW model, where $\rho(z)=\rho_{0}(1+z)^{3}$ (dashed line). The parameters of the GV model are: radius $2.96 \mathrm{Gpc}$ and density contrast 4.05. The same model is presented in Fig. 1; for details of how the model is specified and null gedesics solved see Bolejko \& Wyithe 2009.

existence of a giant void with us at the centre was created by arbitrarily limiting the generality of the model. With its free functions fitted to $\Lambda \mathrm{CDM}$ features rather than to expectations, the giant void does not necessarily follow. Rather, one alternative is that the graph of the density smoothed out over angles around us has the shape of a shallow and wide valley on top of a giant hump.

This giant hump may be a feature of the particular L-T model that we ended up with. Variations in the $\Lambda \mathrm{CDM}$ parameters would modify the details, and the observational constraints we considered are not very tight. Future calculations with other constraints may favor a still different profile at $t=n o w$. Hence we do not wish our paper to become a starting point of a new paradigm in observational cosmology, aimed at detecting the hump. Before this happens, we must decide at the theoretical level whether the hump is a necessary implication of L-T models properly fitted to other observations.

It must be stressed that this hump is not directly observable. It exists in the space $t=n o w$, of events simultaneous with our present instant in the cosmological synchronisation, i.e. it is in a space-like relation to us. This is also the case of the giant void (see, e.g., Fig. 4 of Alnes et al. 2006; Fig. 1 of García-Bellido $\&$ Haugbølle 2008a; Figs. 4 and 6 of Yoo et al. 2008). However, an observational test of the giant void is easier to complete. The reason is that the models considered in this paper have a redshiftspace mass-density almost the same as in the $\Lambda$ CDM model (see Figs. 8 and 18) and as can be seen from Fig. 20, their $\rho(z)$ scales with the redshift in almost the same manner as in the FLRW model, i.e. $\sim(1+z)^{3}$. This is not the case of the giant void ${ }^{5}$ which does not reproduce these features on the past light cone (see Figs. 1 and 20). Thus, unlike the giant void, the giant hump is not observable in $\rho(z)$ or in the number count data.

What is the cause of this difference between the density distribution on our past light cone and in the $t=n o w$ space? It is the oft-forgotten basic feature of the L-T model (and in fact of all inhomogeneous models, also those not yet known explicitly as solutions of Einstein's equations): on any initial data hypersurface, whether it is a light cone or a $t=$ constant space, the density and velocity distributions are two algebraically independent

5 The giant void used here is Bolejko \& Wyithe (2009)'s model with radius of $2.96 \mathrm{Gpc}$ and density contrast of 4.05 . The redshift-space mass-density for this model is presented in Fig. 1. 
functions of position. Thus the density on a later hypersurface may be quite different, since it depends on both initial functions. Whatever initial density distribution we observe can be completely transformed by the velocity distribution. For example, as predicted by Mustapha \& Hellaby (2001) and explicitly demonstrated by Krasiński \& Hellaby (2004), any initial condensation can evolve into a void and vice versa. In FLRW models, there are no physical functions of position, and all worldlines evolve together. Thus, while dealing with an L-T (or any inhomogeneous) model, one must be cautious when applying the Robertson-Walker-inspired prejudices and expectations.

The use of oversimplified L-T models can create another false idea and false expectation. The false idea is that there is an opposition between the $\Lambda \mathrm{CDM}$ model, belonging to the FLRW class, and the L-T model or in general, inhomogeneous models: either one or the other could be "correct", but not both. This putative opposition can then give rise to the expectation that more, and more detailed, observations will be able to tell us which one to reject. In truth, there is no opposition. The inhomogeneous models, like for example the L-T model with its two arbitrary functions of one variable are huge, compared to FLRW, families of models that include the Friedmann models as a very simple subcase. The fact, demonstrated in several papers already (see Célérier 2007, for a review), that even a $\Lambda=0 \mathrm{~L}-\mathrm{T}$ model can imitate $\Lambda \neq 0$ in an FLRW model, additionally attests to the flexibility and power of the L-T model. Thus, if the Friedmann models, $\Lambda \mathrm{CDM}$ among them, are considered good enough for cosmology, then the L-T models can only be better: they constitute an exact perturbation of the Friedmann background, and can reproduce the latter as a limit with an arbitrary precision. While future observations, for example the kSZ effect (GarciaBellido \& Haugbølle 2008b) or the growth of linear structure (Clarkson et al. 2009) will provide a sufficient insight to test particular configurations (like for example a giant void model), we will never be able to reject inhomogeneous models. After all, the Universe as it is, is inhomogeneous. Nowadays we use homogeneous models just for simplicity, and although they have worked well so far, in future they will certainly be replaced by more sophisticated models, either by exact solutions, or what is more probable in light of increasing computation power of computers, by numerical simulations.

When considering models that go beyond the FLRW approximation, one may ask either "what limitations on the arbitrary functions in the models do our observations impose", or "which model best describes a given situation: a homogeneous FLRW model or an inhomogeneous one?" The latter of these questions has often been asked in the context of comparing L-T models without $\Lambda$ to FLRW models with $\Lambda$, and is of much interest for understanding the necessity of introducing $\Lambda$ into the observer's standard cosmological model. Such hypothesis testing questions are often posed in cosmology, but are difficult to address in the current context as they require artificially limiting the generality of the models in question (in order to have a finite number of parameters, so that the test can be performed). Given the lack of motivation for exactly how to perform such a limitation, one is then left in the undesirable circumstance of (potentially) dismissing particular L-T models, while being left with an infinite number of remaining L-T models to evaluate. We therefore prefer to consider the former question. In order to reasonably answer this for the L-T model, a general framework for interpreting observations in the L-T geometrical background should be created (and in the future it should be transformed into a framework for interpreting the observations in a still more general, or the most general geometrical background). Such a program is still in its infancy, but is being actually developed by C. Hellaby and coworkers under the name "Metric of the Cosmos" (Lu \& Hellaby 2007; McClure \& Hellaby 2008; Hellaby \& Alfedeel 2009).

Acknowledgements. M.N.C. wants to acknowledge interesting discussions with Rocky Kolb, Luca Amendola, Roberto Sussman and Miguel Quartin. We also acknowledge useful comments on earlier versions of this text by Charles Hellaby. The work of AK was partly supported by the Polish Ministry of Higher Education grant N202 104838 .

\section{References}

Alexander, S., Biswas, T., Notari, A., \& Vaid, D. 2009, JCAP, 0909, 025 Alnes, H., \& Amarzguioui, M. 2007, Phys. Rev. D, 75, 023506

Alnes, H., Amarzguioui, M., \& Grøn, Ø. 2006, Phys. Rev. D, 73, 083519 Apostolopoulos, P. S., Brouzakis, N., Tetradis, N., et al. 2006, JCAP, 0606, 009 Biswas, T., \& Notari, A. 2008, JCAP, 0806, 021

Bolejko, K. 2008, PMC Phys. A, 2, 1

Bolejko, K. 2009, GRG, 41, 1737

Bolejko, K., \& Wyithe, J. S. B. 2009, JCAP, 0902, 020

Bolejko, K., Krasiński, A., \& Hellaby, C. 2005, MNRAS, 362, 213

Bondi, H. 1947, MNRAS, 107, 410

Brouzakis, N., Tetradis, N., \& Tzavara, E. 2007, JCAP, 0702, 013

Buchert, T. 2000, GRG, 32, 105

Buchert, T. 2001, GRG, 33, 1381

Célérier, M. N. 2000, A\&A 353, 63.

Célérier, M. N. 2007, New Adv. Phys., 1, 29

Clarkson, C., Clifton, T., \& February, S. 2009, JCAP, 0906, 025

Clifton, T., Ferreira, P. G., \& Zuntz, J. 2009, JCAP, 0907, 029

Ellis, G. F. R. 1971, Proceedings of the International School of Physics Enrico Fermi, Course 47: General Relativity and Cosmology, ed. R. K. Sachs (New York and London: Academic Press), 104; reprinted, with historical comments, in 2009, GRG, 41, 581

Enqvist, K., \& Mattsson, T. 2007, JCAP, 0702, 019

Etherington, I. M. H. 1933, Phil. Mag. VII, 15, 761; reprinted, with historical comments, in 2007, GRG, 39, 1055

García-Bellido, J., \& Haugbølle, T. 2008a, JCAP, 0804, 003 García-Bellido, J., \& Haugbølle, T. 2008b, JCAP, 0809, 016 García-Bellido, J., \& Haugbølle, T. 2009, JCAP, 0909, 028 Hellaby, C., \& Alfedeel, A. H. A. 2009, Phys. Rev. D, 79, 043501 Iguchi, H., Nakamura, T., \& Nakao, K. 2002, Prog. Theor. Phys., 108, 809 Kowalski, M., Rubin, D., Aldering, G., et al. 2008, ApJ, 686, 749 Krasiński, A., \& Hellaby, C. 2004, Phys. Rev. D, 69, 023502

Lemaître, G. 1933, Ann. Soc. Sci. Bruxelles A53, 51; English translation, with historical comments: 1997, GRG, 29, 637

Lu, T. H. C., \& Hellaby, C. 2007, CQG, 24, 4107

Marra, V., Kolb, E. W., Matarrese, S., et al. 2007, Phys. Rev. D, 76, 123004

McClure, M. L., \& Hellaby, C. 2008, Phys. Rev. D, 78, 044005

Mustapha, N., \& Hellaby, C. 2001, GRG, 33, 455

Mustapha, N., Hellaby, C., \& Ellis, G. F. R. 1997, MNRAS, 292, 817

Mustapha, N., Bassett, B. A. C. C., Hellaby, C., et al. 1998, CQG, 15, 2363

Pascual-Sánchez, J. F. 1999, Mod. Phys. Lett. A, 14, 1539

Plebański, J., \& Krasiński, A. 2006, An Introduction to General Relativity and Cosmology (Cambridge University Press)

Ruban, V. A. 1968, Pis'ma v Red. ZhETF, 8, 669; English translation: Sov. Phys. JETP Lett., 8, 414 (1968); reprinted, with historical comments: 2001, GRG, 33,363

Ruban, V. A. 1969, Zh. Eksper. Teor. Fiz., 56, 1914; English translation: Sov. Phys. JETP, 29, 1027 (1969); reprinted, with historical comments: 2001, GRG, 33, 375

Silk, J. 1977, A\&A, 59, 53

Schneider, J., \& Célérier, M. N. 1999, A\&A, 348, 25

Simon, J., Verde, L., \& Jimenez, R. 2005, Phys. Rev. D, 71, 123001

Tolman, R. C. 1934, Proc. Nat. Acad. Sci. USA, 20, 169; reprinted, with historical comments: 1997, GRG, 29, 931

Yoo, C. M., Kai, T., \& Nakao, K.-I. 2008, Prog. Theor. Phys., 120, 937

Zibin, J. P., Moss, A., \& Scott, D. 2008, Phys. Rev. Lett., 101, 251303 